\title{
Des catégories aux hypothèses : la théorisation progressive
}

\section{Claude Grignon}

\section{(2) OpenEdition}

1 Journals

Édition électronique

URL : http://journals.openedition.org/ress/133

DOI : $10.4000 /$ ress. 133

ISSN : 1663-4446

Éditeur

Librairie Droz

\section{Édition imprimée}

Date de publication : 1 septembre 2008

Pagination : 55-73

ISBN : 978-2-600-01242-3

ISSN : 0048-8046

\section{Référence électronique}

Claude Grignon, "Des catégories aux hypothèses : la théorisation progressive », Revue européenne des sciences sociales [En ligne], XLVI-142 | 2008, mis en ligne le 01 septembre 2011, consulté le 10

décembre 2020. URL : http://journals.openedition.org/ress/133; DOI : https://doi.org/10.4000/ress. 133

(c) Librairie Droz 


\section{CHAPITRE III}

\section{DES CATÉGORIES AUX HYPOTHÈSES : LA THÉORISATION PROGRESSIVE}

\section{LA QUESTION DU RÉALISME}

Le rôle que jouent les vérités de fait, les assertions d'existence dans les analyses qui précèdent conduit à s'interroger sur le statut épistémologique de la réalité. Dans le débat classique, en philosophie des sciences, entre réalisme et instrumentalisme (ou phénoménisme), nous nous retrouvons ainsi du côté des réalistes $^{1}$, pour qui la science a pour but de «décrire le mécanisme réel de la nature », la «description objective d'une réalité plus fondamentale et plus réelle que la réalité observable» (Bouveresse 2003: 15-47) $)^{2}$, de «décrire de mieux en mieux la réalité profonde de l'univers, par delà le sensible» (Boyer 2000) ${ }^{3}$, alors que pour les instrumentalistes elle doit se contenter de «'sauver les phénomènes' de la façon la plus simple, la plus élégante et la plus commode possible » ${ }^{4}$, de produire des instruments de calcul et de prédiction efficaces, de «classer les phénomènes », de nous donner «une maîtrise et un contrôle de notre environnement empirique $»^{5}$. Pour les anti-réalistes notre sentiment de la réalité, notre conviction que celle-ci existe en dehors de nous et indépendamment de nous résultent directement de notre perception et de notre expérience pratique du monde; quand on passe à une autre échelle, on s'aperçoit que les catégories sur lesquelles repose cette croyance ne sont pas universelles. Mais les sciences de l'homme ne sont pas concernées par cette critique du réalisme. Les phénomènes auxquels elles ont affaire et qu'elles s'efforcent de décrire, les rapports et les régularités qu'elles tentent d'établir, les entités théoriques qu'elles essaient d'élaborer ne se situent pas en effet au niveau microscopique du monde physique. Les différences entre les ordres de réalité sont aussi des différences d'échelle. Il n'existe pas, en sociologie, en ethnologie, en histoire, ni même en économie, d'équivalent de la biologie moléculaire ou de la physique quantique; l'individu (et pourquoi pas, au niveau «sub-individuel», les différentes composantes du moi ou de la personnalité) n’y sont jamais que des métaphores des particules élémentaires.

1 Cf. J. Bouveresse, «Une épistémologie réaliste est-elle possible?» in J.-P. Changeux, La vérité dans les sciences, Paris, O. Jacob, pp. 15-47, et A. Boyer, «Philosophie des sciences », in P. Engel, éd., Précis de philosophie analytique, Paris, PUF, 2000.

2 J. Bouveresse, op. cit.

A. Boyer, op. cit.

4 J. Bouveresse, op. cit.

5 A. Boyer, op. cit. 
Pour les sciences de l'homme, la question du réalisme scientifique, telle qu'elle se pose à la physique et telle qu'on la pose à partir des résultats de celle-ci, n'est donc pas pertinente; vouloir qu'elles se la posent, leur imposer de se la poser de cette manière, c'est se placer et les placer une fois de plus dans une perspective réductionniste. Par ailleurs la question de savoir si les entités théoriques que les sciences constituent sont seulement des constructions, des représentations de l'esprit humain, ou si elles existent indépendamment de celui-ci, est un luxe que les sciences expérimentales peuvent s'autoriser, parce qu'elles rencontrent directement et à volonté la résistance et la sanction d'une réalité sur laquelle elles permettent d'agir; il n'en va pas de même pour les sciences de l'homme.

Parce qu'elles sont moins contraintes, dans la pratique, d'être réalistes, les sciences de l'homme sont, plus que les sciences de la nature, dans l'obligation de faire le «pari réaliste», i. e. de parier sur la possibilité, pour «l'esprit», de «trier, entre ses représentations, celles qui n'ont qu'une valeur verbale ou logique et celles qui restituent l'ordre des choses » (Saint-Sernin 2002: 124) ${ }^{6}$. Pour les sciences de l'homme, l'alternative au réalisme n'est pas l'instrumentalisme, mais le conventionnalisme et sa conséquence, le relativisme. Faute d'avoir suffisamment avancé dans l'élaboration de fictions théoriques vraiment utiles, de classements vraiment commodes ${ }^{7}$, elles n'en sont pas encore à devoir se contenter de «sauver les phénomènes». «Le mérite essentiel de la théorie» est sans doute «de nous révéler des analogies, des parentés et des affinités entre les phénomènes, en particulier entre des phénomènes qui sont à première vue très éloignés les uns des autres et qu'elle s'efforce avec succès de ramener à une unité toujours plus grande », comme l'écrit J. Bouveresse. C'est l'ambition de Darwin et sa définition de la théorie ${ }^{8}$, et ce n'est sans doute "pas la même chose que de lui reconnaître la capacité d'atteindre en profondeur la réalité unifiée qui est sous-jacente aux phénomènes »; mais cette réussite instrumentaliste est rarement atteinte par les sciences de l'homme, que l'interprétation relativiste des révolutions de la physique moderne expose à rester en deçà de la révolution baconienne. Il leur faut donc revenir au programme durkheimien; pour naturaliser les faits humains, en particulier les faits sociaux, historiques, ethnologiques, économiques, il faut les traiter «comme des choses», extérieures à l'esprit, et non comme des idées (Durkheim 1960 [1894]).

On ne revient pas pour autant à un «chosisme universel» qui ne serait «qu'une version assez naïve du réalisme» (D'Espagnat 2002: 48) ${ }^{10}$; on ne se propose pas

${ }^{6}$ B. Saint-Sernin, «Les philosophies de la nature», in D. Andler, A. Fagot-Largeault, B. SaintSernin, Philosophie des sciences, op. cit., p. 124.

Sur les rapports entre commodité et vérité (la commodité comme «marque de la vérisimilitude élevée»), cf. J. Bouveresse, op. cit.

${ }^{8}$ Cf. supra, introduction.

9 «La chose s'oppose à l'idée comme ce que l'on connaît du dehors à ce que l'on connaît du dedans. Est chose tout objet de connaissance qui n'est pas naturellement compénétrable à l'intelligence, tout ce dont nous ne pouvons nous faire une notion adéquate par un simple procédé d'analyse mentale, tout ce que l'esprit ne peut arriver à comprendre qu'à condition de sortir de lui-même (...) Traiter des faits d'un certain ordre comme des choses, ce n'est donc pas les classer dans telle ou telle catégorie du réel; c'est observer vis-à-vis d'eux une certaine attitude mentale.» E. Durkheim, Les règles de la méthode sociologique, op. cit., préface de la $2^{\mathrm{e}}$ édition.

${ }^{10}$ B. d'Espagnat, Traité de physique et de philosophie, Paris, Fayard, 2002, p. 48. 
«d'atteindre les choses elles-mêmes, comme le pensent les dogmatistes naïfs » (Poincaré 1968: 25) ${ }^{11}$. Pour sortir de la spéculation et de l'interprétation libre, il faut à la fois établir les faits et reconstituer, découvrir «les rapports entre les choses». L'opposition entre «les lois phénoménologiques» qui «sont effectivement vraies des objets dans la réalité - ou pourraient l'être» et «les lois fondamentales » qui «sont vraies uniquement des objets dans le modèle» (Cartwright 1983: 4) ${ }^{12}$ renvoie sans doute à deux tendances et à deux définitions opposées du savoir scientifique: «Poincaré remarquait qu'on ne peut pas dire actuellement si la science va aller vers toujours plus d'unité et de simplicité ou, au contraire, vers toujours plus de variété et de complication» (Bouveresse 2003: 23) ${ }^{13}$. Mais l'intelligibilité et la connaissance des contenus ne vont pas l'une sans l'autre. Qu'elle s'appuie sur la modélisation ou sur la reconstitution narrative, l'explication scientifique repose sur la mise en relation, qui est le principe même de la méthode comparative. Le savoir scientifique ne se réduit pas à un catalogue, à un inventaire toujours plus détaillé mais jamais complet de «l'ameublement» de l'univers, particulièrement pléthorique, désordonné et hétéroclite dans le cas des sciences de l'homme. Celles-ci ne peuvent se contenter d'un réalisme des contenus qui les condamnerait aux tâches aveugles et interminables de la description pure, voire à revenir, sous le couvert du sérieux scientifique, à l'histoire anecdotique, à l'ethnographie pittoresque et à la sociographie amusante. Mais par ailleurs on cède à la tentation spéculative ou à la dérive formaliste quand on exclut les contenus pour se fixer sur les relations; on n'explique pas, parce qu'on n'explique rien, si l'on ne sait en quoi «consistent» les objets et ce que désignent les termes que l'on met en rapport.

\section{LES ARTICULATIONS DE LA RÉALITÉ}

Pour rendre la réalité intelligible, il faut y introduire des discontinuités. On retrouve l'opposition entre une volonté descriptive et narrative qui entend «coller» à la réalité et une volonté modélisatrice, qui entend s'en abstraire et la simplifier. Mais il faut noter que ce sont les observateurs les plus confrontés à la diversité et à la profusion des phénomènes, comme les biologistes de l'évolution, qui sont les plus désireux d'introduire des discontinuités, ou plutôt de retrouver, derrière la confusion des apparences, les articulations de la réalité. Pour E. Mayr, la réalité est elle-même discontinue. "Même les peuples primitifs ont des noms pour désigner les différentes sortes d'oiseaux, de poissons, de fleurs ou d'arbres, et ils reconnaissent exactement les mêmes espèces que les taxinomistes modernes. La possibilité de nommer ainsi n'existe que parce que la diversité de la nature n'est pas continue, mais consiste en entités discrètes, séparées par des

\footnotetext{
${ }^{11}$ H. Poincaré, La Science et l'Hypothèse, Flammarion, 1968, p. 25 (cité par J. Bouveresse, op. cit.).

${ }^{12}$ N. Cartwright, How the Laws of Physics Lie, Oxford, Clarendon Press, 1983, p. 4, cité par J. Bouveresse, op. cit. Cf. aussi Buffon: «La seule et vraie science est la connaissance des faits, l'esprit ne peut pas y suppléer». Discours introductif à l'Histoire Naturelle, cité par A. Fagot-Largeault, «La recherche étiologique, chemins causals, histoires, influences, mécanismes, ontologie causale», art. cit.

13 J. Bouveresse, op. cit., p. 23.
} 
discontinuités. On trouve dans la nature non seulement des individus, mais des 'espèces', c'est-à-dire des groupes d'individus qui partagent certaines caractéristiques communes » (Mayr 1989: 247) ${ }^{14}$.

On trouve une idée semblable chez F. Simiand, pour qui le sociologue doit veiller à ce que ses abstractions «se modèlent sur la complexité concrète, respectent les articulations du réel, expriment quelque chose à la fois de distinct et de vrai par rapport à la multiplicité des cas individuels à laquelle elles correspondent » (Simiand 1922: 29, 30) ${ }^{15}$. Toute la question, toute la difficulté est en effet de faire, dans les sciences de l'homme les plus généralisantes, les «bonnes abstractions », celles qui retrouvent les discontinuités du réel. Comme le remarque F. Simiand, c'est le problème auquel le sociologue statisticien est confronté quand il doit définir des variables, et, à l'intérieur de chaque variable des positions pour traiter des données déjà pré-découpées par le questionnaire; c'est aussi celui que rencontre l'ethnologue qui prépare son guide d'observation directe et l'améliore progressivement, au contact du terrain. Mais ce qu'on entend par «réalité » et par «articulations de la réalité » varie selon les sciences. Pour le sociologue, les articulations de la réalité sociale correspondent d'abord aux divisions de la société. C'est l'objet de la morphologie sociale, dans laquelle Durkheim et les durkheimiens (notamment Halbwachs) voyaient un thème d'étude majeur. La morphologie sociale s'apparente pour une part aux sciences du constat, comme la géographie physique ou l'anatomie descriptive; elle se propose d'entreprendre l'inventaire de la diversité sociale, de décrire les groupes dont se compose une société, de repérer leurs positions respectives, de suivre le tracé, plus ou moins net, de leurs frontières. A cette cartographie de «l'espace social» il faut joindre l'exploration du temps, l'étude des articulations chronologiques, des rythmes sociaux.

Mais on ne peut constater l'existence de ces divisions de fait sans remonter à leurs principes, sans énoncer les critères en fonction desquels elles se forment, et selon lesquels les individus se répartissent, se classent et sont classés dans les

${ }^{14}$ E. Mayr, Histoire de la biologie, op. cit., p. 247. A l'inverse, ce sont les naturalistes venus des mathématiques et de la physique, héritiers de la tradition nominaliste, qui ont le plus résisté à l'idée de faire disparaître la singularité des individus (au demeurant interchangeables) en les regroupant et en les rangeant dans des classes. Linné et les linnéens «mettent en avant la discontinuité, Buffon la continuité. Linné adhérait à la philosophie de Platon et à la logique thomiste, tandis que Buffon était influencé par Newton, Leibniz et les nominalistes ». «La nature ne connaît pas d'espèces, genres ou autres catégories déclare Buffon, elle ne connaît que des êtres individuels (...) pourquoi disséquer et détruire cette unité en la décomposant en espèces, genres ou autres catégories?». Id., p. 183.

15 «N'est-ce pas, en effet, un lieu commun de la méthodologie courante que de montrer que le fait scientifique des sciences de la nature, étant (...) détaché, séparé (par définition même, on peut le dire) de la complexité que présente la réalité concrète, est, à proprement parler une abstraction? Mais (...) ce n'est pas à dire que ce détachement, cette séparation, cette abstraction se fasse à la fantaisie de l'expérimentateur, que le fait scientifique soit une entité librement créée par l'esprit du savant, à la manière des entités de la scolastique médiévale. Pour mériter ce nom de fait scientifique, pour entrer dans la science, il faut que cette abstraction, tout en se distinguant de la complexité concrète, se modèle cependant suffisamment sur elle, respecte, comme l'a dit un philosophe contemporain, les articulations de la réalité (...)». F. Simiand, Statistique et Expérience, remarques de méthode, op. cit., pp. 29, 30. Rappelons au passage les plaisanteries de Simiand, - essentialiste? - sur les «plaisanteries nominalistes trop faciles ». F. Simiand, «Méthode historique et sciences sociales », art. cit. 
différents groupes (selon le sexe, selon l'âge, selon le lieu de naissance ou d'habitation, selon la profession, selon le revenu, etc.). Ces critères (on aura reconnu dans les exemples qui précèdent les variables qui constituent le «talon» des enquêtes statistiques) sont par définition discutables; les plus objectifs, ceux qui reposent sur les caractéristiques les plus factuelles, comme le sexe ou l'âge, ne sont jamais tout à fait neutres, dans la mesure où ils sont des instruments et des enjeux de classement social, au même titre que les catégories savantes qu'ils engendrent. Celles-ci sont doublées par des catégories et des oppositions indigènes, plus ou moins élaborées, plus ou moins renseignées par l'expérience, plus ou moins bornées par un Umwelt plus ou moins restreint, entre jeunes et vieux, entre hommes et femmes, entre «Eux» et «Nous», entre riches et pauvres, entre parisiens et provinciaux, ou encore entre intellectuels et manuels. Ces représentations indigènes font partie de la réalité sociale; elles contribuent à renforcer les oppositions et les divisions dont elles sont l'expression. Il faut donc les inclure dans le tableau des articulations de la réalité; mais le sociologue se gardera de les confondre avec les catégories qu'il s'efforce d'élaborer et de mettre en œuvre.

Pour faire des abstractions réalistes, il faut, pour commencer, s'abstenir de sociologiser trop vite et prendre au contraire le temps d'examiner la base matérielle des faits sociaux, se donner la peine de connaître les conditions et les contraintes physiques, techniques, biologiques, etc., dans lesquelles apparaissent et se développent les formations sociales que l'on étudie; leur importance est sans doute inégale selon les domaines, mais on risque toujours, si on les néglige, de simplifier abusivement, de schématiser et de théoriser à la faveur de l'ignorance. Il faut, dans le même esprit, rechercher les indicateurs de division les plus objectifs, comme par exemple, s'agissant du cycle de vie, l'âge de la scolarité obligatoire, l'âge de la retraite, l'âge moyen au premier emploi, ou, s'agissant des rythmes sociaux, la durée légale du travail, les dates des vacances scolaires, les horaires d'ouverture des magasins, les horaires et la durée des repas, etc. On peut également s'appuyer sur les indicateurs de discontinuité entre des niveaux hiérarchiques qu'on trouve dans l'organisation des institutions ou dans les dispositions légales relatives à la définition des professions, des «corps» professionnels et des grades, par exemple la hiérarchie des ordres d'enseignement, l'opposition entre sous-officiers et officiers, entre techniciens et ingénieurs, entre infirmières et médecins. On sera particulièrement attentif aux barrières qui interdisent de changer de niveau, comme l'absence de diplôme ou l'ignorance de la langue (qui enferme en même temps dans les limites du groupe d'origine), aux mécanismes de sélection et de relégation, notamment scolaires, aux processus cumulatifs, en matière de revenus ou de notoriété, véritables incrémenteurs de distance sociale qui transforment des différences de degré en différences de nature en créant des seuils infranchissables et des ordres séparés.

Il faut enfin essayer de mettre à profit l'ambivalence des faits sociaux pour les redéfinir en partant de leur sens usuel et des notions courantes qui les désignent. En effet ces faits et ces notions sont des entités pratiques, et, pour ainsi dire, des abstractions concrètes. Chacun sait par expérience ce qu'est un salaire, un prix, un emploi, un niveau de revenu, un diplôme, ou encore le chômage, l'héritage, le mariage, la famille; même, chacun «a son idée» d'entités sociales plus abstraites, comme la religion ou la justice. Ces abstractions se manifestent, se matérialisent dans des objets, des documents, des pratiques observables, constatables, 
enregistrables; on peut, conformément aux principes durkheimiens, interviewer des chômeurs, relever des prix, calculer l'endogamie, l'âge moyen à la naissance du premier enfant, les probabilités de divorce, etc. La «résistance» des faits sociaux réside pour une bonne part dans le pouvoir et dans les contraintes qu'ils exercent sur les individus qui les subissent; les sanctions de la réalité se présentent au sociologue par l'intermédiaire des sanctions auxquelles expose le manquement aux usages et aux normes sociales. C'est sur cette base, en partant de leur acception courante, vague mais renvoyant à une multiplicité de pratiques et de contraintes observables, qu'il faut redéfinir et spécifier ces notions pour en faire des concepts sociologiques. C'est ce que fait par exemple Simiand quand il définit le salaire en partant non d'une idée préconçue mais des traits distinctifs qui ne se retrouvent pas dans les autres rétributions (honoraires, solde, émoluments, etc.) avec lesquelles on a tendance à le confondre. Les abstractions concrètes que le sociologue ne cesse de rencontrer jouent ainsi le rôle d'intermédiaires, qui permettent de dégager progressivement des concepts à partir de l'observation, de la description et de la reconstitution des faits. D'un côté elles renvoient à l'expérience de la vie sociale, aux réactions, intellectuelles, morales et affectives que cette expérience suscite, et à leur expression usuelle; de l'autre, une fois retravaillées et redéfinies, elles s'appliquent à des classes de faits, à des ordres de pratiques que le sens commun sépare, qu'il ne viendrait pas à l'idée de rapprocher.

\section{RÉALISME ET LANGAGE NATUREL}

On trouve de bons exemples de cette conceptualisation progressive dans des récits à la fois suggestifs et significatifs, qui donnent à penser en donnant à voir, en stimulant la mémoire et l'imagination, en facilitant les rapprochements et les comparaisons ${ }^{16}$. C'est le cas, par exemple, des notations exactes et justes par lesquelles Richard Hoggart fait comprendre en quoi consiste et ce que signifie l'ambivalence des cultures populaires et des rapports que les intellectuels qui en sont issus entretiennent avec elles, ou, dans un genre moins impressionniste, de la description technique détaillée des ateliers que l'on trouve dans le Guide d'étude directe des comportements culturels de Marcel Maget, vrai tremplin pour l'imagination sociologique (Hoggart 1991; Maget 1962) ${ }^{17}$; ou encore de la manière dont un Mauss ou un Granet trouvent les formules les plus capables de nommer et de rassembler les comportements factuels mentionnés dans les sources. Soit, entre autres, la «scène» saturée en concepts, où M. Granet définit la communion et l'expiation à partir de la reconstitution des bravades à l'aide desquelles s'établit la hiérarchie féodale dans la Chine ancienne:

${ }^{16}$ Je reprends ici les analyses que j’ai développées dans «La formalisation et les sciences du récit », in J-Y Grenier, C. Grignon, P-M. Menger (dir.), Le modèle et le récit, Paris, 2001, Editions de la Maison des Sciences de l'Homme.

${ }^{17}$ R. Hoggart, 33 Newport Street, autobiographie d'un intellectuel issu des classes populaires anglaises, Paris, Gallimard / Le Seuil, coll. Hautes Etudes, 1991; M. Maget, Guide d'étude directe des comportements culturels, Paris, CNRS, 1962. 


\begin{abstract}
Les beuveries féodales, principes de communions inféodantes, commencent par ce geste de défi qu'est l'offre d'une coupe. Avant le combat, on offre une coupe à l'ennemi. Qu'il se réconforte! On lui tiendra tête tout de même! Après la victoire, on offre une coupe au vaincu. C'est le traiter en coupable, car la défaite a révélé en lui un criminel que l'on doit contraindre à se purger d'une vertu mauvaise. Mais c'est encore le réhabiliter en effaçant le passé et c'est aussi éviter un retour de vengeance en proposant une communion. C'est enfin marquer son propre triomphe. Aux tournois du tir à l'arc, les vaincus, tenant leurs arcs débandés, reçoivent des vainqueurs une coupe de corne. Ils boivent et expient leur défaite. Les vainqueurs expient ensuite leur victoire; ils boivent à leur tour, mais dans la coupe qui sert aux investitures. La hiérarchie une fois créée, la solidarité est confirmée: tous communient en buvant à la ronde. Distribuer les coupes, distribuer les honneurs se disent avec la même expression (Granet 1968 [1929]: 257) ${ }^{18}$.
\end{abstract}

Une association aussi étroite, aussi intime, de la conceptualisation et de l'imagination ne va pas sans risques. Elle expose l'observation des faits, directe ou indirecte, à des partis-pris interprétatifs difficilement contrôlables. Le pouvoir d'évocation du langage naturel, qui assure le contact avec la réalité et dont dépendent à la fois le réalisme et l'intelligibilité de la description, suggère constamment à l'observateur ou au lecteur des interprétations hasardeuses difficiles à écarter tant elles s'imposent à l'imagination. Trop vivement sollicitée, celle-ci peut conduire les exégètes les plus érudits et les plus scrupuleux à sur interpréter les données ou les textes dont ils disposent (Van Gulik 1971) ${ }^{19}$. Comme le langage naturel tire pour une bonne part son pouvoir d'évocation de sa «souplesse», c'est-à-dire, en fait, de ses anomalies sémantiques, on ne peut pas compter sur lui pour opposer aux divagations de l'imagination le contrôle logique du raisonnement (Gardin $1974)^{20}$. Plus gravement encore, la nécessité d'évoquer et de figurer la réalité pour

${ }^{18}$ M. Granet, La civilisation chinoise, Paris, Albin Michel, L'évolution de l'humanité, 1968 [1929], p. 257.

${ }^{19}$ C'est ce que R. Van Gulik reproche aux sinologues de l'école française, et en particulier à Granet: «Dans sa Civilisation chinoise (pp. 418-419) et plus tard dans ses Catégories matrimoniales (p. 152), M. Granet interprète ce passage et ceux qui concernent les femmes obstinées à demeurer muettes comme une preuve que dans la Chine ancienne il existait des «joutes maritales » qui de tout temps marquèrent les trois premiers mois du mariage. C'est là un des nombreux exemples de la façon dont Granet peut lire dans un texte chinois plus de choses qu'il n'en contient et prend des faits accidentels pour une règle générale. Granet fut l'un des grands sinologues français et ce fut aussi un penseur d'une haute originalité, mais ses œuvres - bien qu'il s'agisse toujours de contributions stimulantes et importantes par leur érudition - s'exposent souvent à la critique à cause de ces deux inclinations ». R. Van Gulik, La vie sexuelle dans la Chine ancienne, Paris, Gallimard, 1971 (traduction de Sexual Life in Ancient China, E.Y. Brill, Leiden, 1961), p. 60, n. 1. (S'agissant du passage précédemment cité, Van Gulik reprend cependant, p. 32, l'interprétation des beuveries féodales donnée par M. Granet).

${ }^{20}$ Cf. J.-C. Gardin, Les analyses de discours, Neuchâtel, Delachaux et Niestlé, 1974, p. 83. «a) des termes LN [langue naturelle] différents sont tenus pour équivalents (synonymies); $b$ ) à un même terme LN sont associés plusieurs sens distincts (homonymies, homographies, polysémies; ex : "circulation", routière, sanguine, monétaire, etc.); $c$ ) des tournures syntaxiques différentes sont tenues pour équivalentes quant à la relation logique sous-jacente (allotaxies, ex.: "action de x sur y", "rôle de x dans y", "y est affecté, influencé, etc., par x", etc.); d) à une même tournure LN sont associées des relations logiques distinctes (homotaxies; ex. : les tournures génitives, à sens tantôt qualificatif -“cancer du poumon"- tantôt causal, "cancer du tabac", etc.); e) des équivalences plus complexes sont posées entre mots et phrases d'un même langage (définitions; ex.: "hypothermie", "température inférieure à la normale", etc.)». 
la rendre intelligible risque de réintroduire dans les sciences de l'homme le mimétisme caractéristique de la pensée magique. Puisqu'il faut peindre pour expliquer, on finit par croire que peindre et expliquer sont la même chose, on confond, comme dit Taine, «l'art» et «l'analyse»:

Peindre, c'est faire voir, et c'est un emploi tout spécial que de faire voir les personnages passés. Si quelqu'un s'y efforçait, il faudrait qu'il eût été préparé à ce travail d'artiste par des études d'artiste; qu'il eût été, dans sa jeunesse, romancier comme Walter Scott, et même poète; qu'à ce titre il aperçût naturellement et de prime-saut les plus légères nuances et les plus fragiles attaches des sentiments; que peu à peu le progrès de l'âge et les reploiements de la réflexion aient ajouté en lui le psychologue à l'artiste; que la finesse française, la délicatesse parisienne, l'érudition du $19^{\mathrm{e}}$ siècle, l'épicurisme de la curiosité, la science de l'homme et des hommes, lui aient composé un tact exquis et unique. (...) Il n'aurait pas assez, pour une telle œuvre, du style simple des logiciens et des classiques. Il aurait besoin de phrases plus enroulées, capables de se tempérer et de s'atténuer les unes les autres, de mots plus spéciaux, traînant avec eux un long cortège d'alliances et de souvenirs. Il faudrait moins le lire que le goûter (...). [Mais] laissez l'objet qui a fourni matière à la peinture fournir matière à la philosophie; permettez à l'analyse de venir après l'art (...). Les deux études diffèrent, puisque l'imagination diffère de l'intelligence, et le raisonnement a le droit de décomposer ce que les yeux ont contemplé et ce que le cœur a senti. Je puis me demander d'où viennent ces qualités, ces défauts, ces passions, ces idées; lesquels sont effets, lesquels sont causes (...). Dès lors, tous vos préceptes tombent; les règles qui gouvernaient la peinture n'ont point de prise sur l'analyse; ce qui serait une faute pour la première devient un devoir pour la seconde. Vous développiez, elle réduit. Vous poursuiviez les détails délicats, elle recherche les grandes causes. Vous saisissiez au vol ces traits fugitifs qui font surgir dans l'imagination toute une figure; elle s'attache à ces forces génératrices qui produisent dans la vie toute une série d'événements. Vous négligiez beaucoup de points qui lui importent; elle néglige beaucoup de points qui vous intéressent. Pour elle le changement d'objet a changé le reste; si l'on trouve son but légitime, on ne peut lui interdire la voie qui la mène à son but (Taine 1858) ${ }^{21}$.

Reste que la sociologie ne peut se passer de la puissance d'évocation du langage naturel pour dégager progressivement ses concepts de l'observation et de la description ${ }^{22}$. Pour rendre intelligible la réalité à laquelle s'intéressent les sciences de l'homme, il faut en effet la représenter, et, pour commencer, se la représenter. Il ne s'agit pas seulement, pour le sociologue ou l'historien, d'être intelligible pour les autres, d'évoquer ce qui est connu pour que le lecteur puisse reconnaître ce dont il est question; si la réflexion et la pensée abstraite, dans nos disciplines, se font en langage naturel, c'est parce qu'elles ne peuvent se passer de l'imagination, qui permet d'identifier ce que l'on observe et de contrôler le réalisme des désignations, des contenus et des découpages. Il faut donc essayer de concilier l'usage, obligatoire, du langage naturel avec les exigences d'exactitude, de clarté, d'impersonnalité et de concision qui caractérisent l'écriture scientifique, et qui, le plus souvent, l'opposent à l'écriture littéraire. Passer de l'écriture littéraire à l'écriture scientifique, c'est passer à un niveau du langage naturel plus

${ }^{21}$ H. Taine, Essais de critique et d' histoire, Paris, Hachette, 1858, pp.V sq.

${ }^{22}$ L'apport de la force évocatoire du langage naturel à la conceptualisation se voit dans la fabrication des outils: les bons questionnaires, les bons guides d'entretien sont riches en termes intermédiaires et ambivalents, capables à la fois d'intéresser l'enquêté parce qu'ils lui «disent quelque chose » et de prendre ou de recevoir un sens savant pour le sociologue. 
écrit, plus détaché de l'oral. Le langage des gestes (l'action oratoire, les mimiques de l'avocat, de l'homme politique, mais aussi bien du professeur) accompagne et soutient constamment le langage oral. Il survit dans les effets rhétoriques de la langue écrite, dans la poésie et dans le roman, mais aussi dans l'essayisme et dans les «discours», qui cherchent à emporter la conviction en invitant le lecteur, au sens propre, à s'émouvoir, à ébaucher en pensée des gestes, des connexions motrices $^{23}$. Il faut neutraliser ces effets («tordre son cou à l'éloquence») pour observer, comparer et classer en langage naturel, et construire ainsi, conformément à la démarche théorique des sciences de l'homme généralisantes, des entités de plus en plus abstraites et en même temps de plus en plus chargées de contenus, capables de désigner et de rassembler des faits nombreux et hétérogènes; par exemple définir le sacrifice, distinguer la religion de la magie, etc.

\section{TAXINOMIE ET TYPOLOGIE}

La généralisation s'appuie en effet aussi sur le classement ${ }^{24}$. On s'élève progressivement du particulier au général (et du concret à l'abstrait) en réunissant des objets et des événements divers entre lesquels on décèle des ressemblances; sur la base de ces regroupements, auxquels on attribue des noms, on forme des catégories dans lesquelles on s'efforce de ranger d'autres objets singuliers, d'autres individus (ce qui met ces catégories à l'épreuve et contraint éventuellement à les remanier ou à les abandonner). La constitution des classes, et l'attribution des spécimens à une classe (détermination) se font par comparaison: comparaison des spécimens entre eux, et, éventuellement, comparaison des spécimens à un type. C'est cette démarche que Simiand propose d'appliquer à la «réalité historique $»$ :

La réalité historique est complexe. Il y a des différences entre des phénomènes analogues rencontrés en des sociétés et à des époques diverses; tout cela n'est pas nié par nous, mais n'entame pas la légitimité ni des études comparatives ni des constitutions de types, si elles sont faites avec la critique qui convient (Simiand 1906a) ${ }^{25}$. Ce n'est pas s'éloigner du réel et se jeter dans des constructions logiques et artificielles que d'analyser ce réel avec des catégories claires, mais précises, et que d'y chercher des relations à forme générale, bien que toujours proches de la donnée de fait, car seules ces catégories et ces rela-

${ }^{23}$ Plus un texte est «littéraire», et plus il est difficile à traduire. C'est que la langue littéraire, en particulier la poésie, parle aux affects et que les affects varient selon les cultures. Cf. Wilhelm von Humbolt, Sur le caractère national des langues, Paris, Seuil, Points/Essais, 2000, traduction de D. Thouard, pp. 29, 30 [article de 1799, Gesammelte Schriften, III, 3]: «Il n'y a donc que l'enthousiasme qui soit capable de réveiller et de maîtriser l'imagination, et c'est au poète de l'inspirer. C'est peut-être là la raison pour laquelle il est impossible de sentir entièrement un poète étranger. L'enthousiasme se compose d'une infinité de rapports que les objets ont avec nos sentiments et avec notre caractère; et il faut être élevé dans l'habitude d'une langue, avoir pensé et senti avec elle, pour que chaque phrase et chaque mot se présentent à nous avec toutes ses nuances, qu'il réveille tous les souvenirs capables de renforcer l'idée qu'il nous offre».

24 «Les classifications sont nécessaires partout où l'on a affaire à la diversité». E. Mayr, Histoire de la biologie, op. cit., p. 152.

${ }^{25}$ F. Simiand, «Compte rendu de G. Salvioli, Le capitalisme dans le monde antique», in L'Année sociologique, Vol. X, 1906, pp. 532-534. 
tions donnent une intelligence véritable de la complexité même de ce réel (Simiand $1906 b)^{26}$.

Mais faut-il, pour généraliser, constituer des types, comme le propose Simiand? Ces types sont-ils des spécimens réels, ou des modèles, des fictions idéales? Les classifications sont-elles des typologies ou des taxinomies? Ces questions se sont d'abord posées aux naturalistes. L'histoire de la systématique est traversée, depuis Aristote, par l'opposition entre deux façons de classer les animaux et les plantes, selon que l'on considère les espèces comme des types ou comme des populations. Comme biologiste, Aristote «forme des groupes sur la base de l'observation »; son ouvrage est «entièrement bâti sur des comparaisons: de structure (anatomie comparée), de biologie reproductive et de comportement »; comme philosophe, "père de la scolastique», il superpose à cette "approche phénétique pleine de bon sens (...) un système d'évaluation des attributs » spéculatif, fondé sur la hiérarchie des quatre éléments (Mayr 1989: 156, 157) ${ }^{27}$.

Assimiler les catégories à des types, les définir par un nombre de caractèresclés, i.e. d'attributs, d'autant plus réduit qu'elles occupent un rang plus élevé dans la hiérarchie des classes, c'est donc revenir à la classification descendante, obtenue par division logique et dichotomisations successives, à laquelle aboutit le système d'Aristote ${ }^{28}$; c'est se rattacher à une tradition essentialiste elle-même ambivalente, dans la mesure où elle invite à un découpage spéculatif arbitraire de la réalité ${ }^{29}$, mais où elle postule, contre le nominalisme, que celle-ci est discontinue, divisée et «pré-découpée ${ }^{30}$. On trouve en sociologie une opposition

${ }^{26}$ F. Simiand, «Compte-rendu de P. Mantoux, La Révolution industrielle au XVIII siècle», in L'Année sociologique, Vol. X, 1906, pp. 539-551.

${ }^{27}$ E. Mayr, Histoire de la biologie, op. cit., pp. 156, 157.

${ }^{28}$ La classification descendante finira par être remplacée par «la classification ascendante par formation empirique de groupes » qui est aujourd'hui la méthode «employée par tous les taxinomistes, au moins dans les premiers stades d'une classification». «L'adoption d'une procédure de classification par 'inspection' représenta une révolution méthodologique complète. La direction des premières étapes classificatoires ne se trouvait pas seulement inversée, des principes tels que le recours à un seul caractère à chaque étape de division (fundamentum divisionis) se voyaient bouleversés. Désormais, on prenait en considération de nombreux caractères, voire 'tous les caractères', comme certains des partisans de la classification ascendante l'ont proposé» E. Mayr, Histoire de la biologie, op. cit., pp. 192, 194.

${ }^{29}$ Sur l'essentialisme sous-jacent à la notion de type, voir E. Mayr, op. cit., p. 251: «La présence d'une même essence [eidos] était inférée sur la base d'une similitude. Les espèces étaient donc simplement décrites comme des ensembles d'individus similaires, lesquels étaient différents des individus appartenant à d'autres espèces. Les espèces, ainsi conçues, représentaient différents 'types' d'organismes. Les individus, en vertu de ce concept, n'avaient pas de rapports particuliers entre eux; ils étaient l'expression du même eidos. Toute variation résultait d'une manifestation imparfaite de l'eidos.»

30 «Pour le nominaliste, seuls les individus existent, tandis que les espèces, ou toute autre 'classe', ne sont que des abstractions construites par l'homme». E. Mayr, op. cit., p. 258. Le terme de catégorie a été utilisé dans deux sens différents jusqu'à l'introduction du terme de taxon (en 1926). Un taxon est «un objet zoologique ou botanique concret», «un groupe d'organismes de rang taxinomique quelconque, suffisamment distinct pour qu'on puisse le dénommer et l'assigner à une catégorie précise (...) les rouge-gorges, les grives, les passereaux, les oiseaux, les vertébrés, les cordés et les animaux, sont des groupes d'animaux réels, ce sont des taxa. Le rang qui est donné aux taxa mention- 
analogue, entre le «nominalisme des historiens» pour qui «les sociétés constituent autant d'individualités hétérogènes, incomparables entre elles », qui rend «toute généralisation à peu près impossible», et le «réalisme extrême » des philosophes, pour qui les événements historiques «n'ont de valeur et d'intérêt que comme illustration des lois générales qui sont inscrites dans la constitution de l'homme», «des attributs généraux de la nature humaine» (Durkheim 1960 [1894]: 76-77) ${ }^{31}$. Durkheim voit dans la notion d'espèce sociale un «intermédiaire » qui permettrait de sortir de cette alternative; mais, s'agissant de constituer ces espèces, il refuse la méthode monographique, qui ajournerait «la sociologie jusqu'à l'époque indéfiniment éloignée où l'histoire, dans l'étude qu'elle fait des sociétés particulières, sera parvenue à des résultats assez objectifs et définis pour pouvoir être utilement comparés », et considère les espèces, ou les genres de société non comme des catégories constituées en regroupant le plus grand nombre possible de faits observés dans des contextes (époques, lieux) différents, mais comme des types, définis par la réunion «d'un petit nombre» de «caractères particulièrement essentiels, soigneusement choisis », caractères-clés, «faits décisifs » ou traits pertinents ${ }^{32}$.

Expression d'une essence dont les individus concrets et singuliers (objets, événements) qui se présentent dans la réalité participent à des degrés divers, d'une perfection idéale dont ils s'approchent plus ou moins sans jamais la réaliser, le type ainsi conçu s'oppose au type réel des systématiciens actuels, qui comparent le spécimen qu'il s'agit de déterminer avec un spécimen existant, choisi et conservé comme référence. C'est le type idéal de Max Weber et, avant Weber, d'Auguste Comte: «Ce juste degré d'exagération qu'exige nécessairement l'indication d'un type quelconque, au-dessous duquel la réalité ne sera jamais que trop maintenue ${ }^{33}$. Par cet essentialisme sous-jacent, l'élaboration des types, dans les sciences de l'homme généralisantes, s'apparente à la modélisation dans les sciences formelles. Comme le triangle ou la sphère de Galilée, le type idéal est une abstraction obtenue par élimination des accidents, c'est-à-dire en distinguant et en séparant l'essentiel de l'accidentel. La constitution empirique des espèces elle-même obéit sans doute, pour une part, au principe de la hiérarchisation des

nés dans la classification hiérarchique est indiqué par les catégories dans lesquelles ils sont placés: espèce, genre, famille, sous-ordre, ordre, classe, sous-embranchement, embranchement et règne ». Id., pp. 248, 208. Les taxa sont donc «vraiment 'naturels' ou 'réels'»; en revanche «la catégorie dans laquelle on range un taxon relève d'une décision arbitraire» (Ernst Mayr ne précise pas si l'arbitraire porte aussi sur les noms donnés aux catégories et sur l'organisation hiérarchique elle-même, nombre de rangs et positions respectives des rangs). En dépit de cette clarification, la réalité des espèces continue de poser problème: les espèces sont-elles des «unités de base de diversité de la nature », représentent-elles «le plus bas niveau de discontinuité réelle au-dessus de l'individu», ou bien sont-elles «de simples constructions théoriques de l'esprit humain?». Id, pp. 246, 247.

${ }^{31}$ E. Durkheim, Les règles de la méthode sociologique, op. cit., p. 76-77.

${ }^{32} \mathrm{Ibid}$, p. 78, 80. A la méthode monographique, dont «on fait remarquer qu'elle seule est recevable dans une science d'observation », Durkheim préfère «la vraie méthode expérimentale », qui tend «à substituer aux faits vulgaires, qui ne sont démonstratifs qu'à condition d'être très nombreux, et qui par suite, ne permettent que des conclusions toujours suspectes, des faits décisifs ou cruciaux, comme disait Bacon». Ibid, p. 79.

${ }^{33}$ Cours de philosophie positive, leçon L (IV, 291). 
caractères ${ }^{34}$; mais, dans la mesure où ils sont construits a priori, les types sociologiques ou historiques risquent encore plus de «s'éloigner du réel» et d'être des types ad hoc, fondés sur une sélection décisoire de traits qui immunise ab initio l'intuition interprétative dont elle est issue, et dont il est en conséquence impossible de mesurer l'arbitraire. Le type se confond alors avec ce que Cournot appelle le genre, qui «ne représente que 'l'abstraction logique', i.e. la simple similitude d'un seul caractère, ou d'un très petit nombre de caractères réunis en un concept chez divers individus, dont nous ne faisons une classe que par la considération décisoire de ce concept $\gg\left(\right.$ Cournot $1975[1851)^{35}$.

L'opposition entre le type idéal et la classification par référence à des spécimens réels renvoie donc à deux manières d'abstraire et de généraliser opposées, d'un côté à la façon des sciences nomothétiques, de l'autre à la façon des sciences de l'observation. On tend pourtant à les confondre, car tout type renvoie à une typologie plus ou moins explicite, qui passe elle-même pour une taxinomie. Ce qui constitue le type, c'est, en effet, la présence et la combinaison de propriétés, c'est-à-dire de traits distinctifs qui le définissent en propre, par opposition aux autres types chez lesquels ces propriétés ne se retrouvent pas. En outre, on finit toujours par passer ou par revenir, plus ou moins vite, plus ou moins tôt, à la démarche top down, à la spéculation et à la déduction; mais plus on prend le temps et la peine d'observer, de collecter les faits, et plus les types idéaux que l'on fabrique sont réalistes, plus ils respectent, retrouvent les articulations de la réalité, plus ils se rapprochent des catégories des naturalistes. Une modélisation rigoureuse, aussi peu dépendante que possible de la théorie qui l'inspire, exigerait au moins que l'on répertorie les traits que l'on décide de négliger. Lorsqu'on peut montrer que les caractères-clés choisis sont effectivement les plus déterminants, que ce sont eux qui expliquent le mieux la présence du plus grand nombre d'autres caractéristiques, le type sociologique, ou historique, se rapproche de la modélisation et du type scientifiques. A l'inverse, lorsque les caractères qui le définissent ont été sélectionnés intuitivement, pour leur pouvoir évocateur, il se rapproche des types littéraires ou philosophiques; le type se confond alors avec le portrait. La ressemblance est assez forte, probablement aussi assez profonde, pour que la

34 «Dans le cas des végétaux, les botanistes avisés savaient que de toutes les parties de la plante, les 'fructifications' (fleurs, fruits, graines) étaient celles qui fournissaient les meilleurs traits diagnostiques.» Adanson (1727-1806) se refuse à classer les caractères en essentiels et accidentels, mais voit «clairement que tous les caractères n'ont pas la même valeur taxinomique (...) Il insista sur deux points: 1- certains caractères n'apportent aucune amélioration au processus de classification, et on ne doit pas s'en occuper; 2- les caractères qui fournissent le plus d'informations diffèrent de famille à famille. Chaque famille a son propre 'génie'». De son côté, la constitution des types repose elle aussi sur le principe de la corrélation des caractères, introduit en biologie par Cuvier. E. Mayr, op. cit., pp. 189, 196, 186.

35 «Au contraire, le véritable type, ou 'abstraction rationnelle' [que Cournot appelle aussi 'genre naturel'] (...) est l'ensemble d'un grand nombre de caractères formant un tout organique et dont la réunion ne peut s'expliquer par le hasard, c'est-à-dire par le concours de causes qui ne seraient point enchaînées et subordonnées les unes aux autres. Ainsi les 'êtres volants' forment un genre artificiel comprenant oiseaux, chéiroptères, insectes, lesquels appartiennent à trois types très différents ». A. A. Cournot, Essai, op. cit., chap. XI, not. \$164, 166, 167; Voir aussi le Traité de l' enchaînement des idées fondamentales dans les sciences et dans l' histoire, Paris, Vrin, 1982 [1861], pp. 51 (type et modèle), 177 (type et genre), 213. 
sociologie emprunte des types à la littérature. C'est le cas par exemple de l'héritier et du boursier: "Barrès a développé de son fond le plus sincère et le plus conscient, pour lui et pour la France, une philosophie d'héritier. Barrès a pensé un secteur de vie nationale sous forme de cette opposition du boursier et de l'héritier» (Thibaudet 1979 [1927]: 129) ${ }^{36}$.

Le succès des types littéraires dépend en grande partie du degré auquel ils reproduisent des stéréotypes sociaux. Le sociologue est lui aussi forcé de composer avec ces stéréotypes, qui font partie de la définition sociale des catégories et des types qu'il s'efforce de définir. Il est tentant de profiter de la perspicacité satirique dont ces stéréotypes sont animés; mais comment neutraliser leur malveillance? Enjeu des affrontements symboliques entre les groupes, ils engagent le sociologue à prendre parti, à reprendre à son compte, plus ou moins ouvertement, les jugements de classe dont chaque classe est la cible; c'est le cas en particulier de la sociologie engagée. Mieux vaut sans doute parler de classes dominantes, voire supérieures ou moyennes, à la rigueur de bourgeoisie, que de bourgeois ou de petits bourgeois; mais cette euphémisation, neutralisation formelle, n'empêche pas de jouer sur les stéréotypes qu'elle recouvre et qu'elle continue d'évoquer. De même l'opposition entre fractions intellectuelles et fractions possédantes réactive l'opposition, héritée de la Bohème romantique, entre l'Artiste ou l'Intellectuel («l'intello») et le Bourgeois («l'Epicier»). Le sociologue doit aussi prendre conscience des censures que lui impose son propre ethnocentrisme de classe et de fraction; c'est le cas, par exemple, de l'interdit qui frappe les stéréotypes visant les dominés et les étrangers, cible d'une tradition lettrée bien établie de réprobation morale. Il faut donc clarifier le plus possible les relations et les ressemblances que les types sociologiques entretiennent avec les stéréotypes sociaux. En quoi, par exemple, l'idéalisation se distingue-t-elle de la caricature? Comme les types idéaux, les stéréotypes accusent des traits considérés comme spécifiques et exemplaires. Ils représentent la réalité en l'exagérant; pour paraître «plus vrais que nature », il leur faut sacrifier l'exactitude à l'impression de vérité. Reste que dans leur cas le choix de ces traits est arbitraire; dicté par les passions sociales, il en dit plus sur les partis-pris de l'auteur que sur l'objet de ces partis-pris.

Il n'est sans doute pas possible de transposer directement aux sciences de l'homme collectif la démarche par laquelle la biologie des espèces dégage ses catégories et ses concepts de l'observation. Les articulations de la réalité ne s'y présentent pas de la même manière, ne s'imposent pas à l'observation avec la même force que dans les sciences de la nature; le sociologue qui cherche à distinguer des classes, l'historien qui cherche à caractériser des âges, des ères ou des époques, l'ethnologue qui veut délimiter et définir des cultures ne trouvent pas l'équivalent de la distinction de fait, «naturelle», entre les animaux et les plantes, entre les arbres et les arbustes, entre les animaux qui volent, les animaux qui

${ }^{36}$ A. Thibaudet, La république des professeurs, Paris-Genève, Slatkine, 1979 [1927], p. 129.

Les types philosophiques évoquent eux aussi des cas concrets. Le tyran de Platon, par exemple, nous fait penser à nos dictateurs; Platon lui-même, quand il fait correspondre la typologie des régimes politiques et celle des tempéraments, pense à Alcibiade ou à la conspiration de Cinadon (République, Livre VIII). Ce pouvoir d'évocation des fictions spéculatives en fait des grilles de lecture infaillibles, impossibles à prendre en défaut. 
marchent et les animaux qui rampent, etc. ${ }^{37}$ Pour que la classification des animaux se développe, «il fallut attendre que l'on cessât de prendre les habitats ou les mœurs comme critères de classification et que l'on se basât sur les données anatomiques » (Mayr 1989: 172) ${ }^{38}$; mais les sociologues sont obligés de fonder leurs classifications sur les différences entre les conditions, les modes et les niveaux de vie. On ne peut pas non plus appliquer aux sciences de l'homme le principe de la classification post-darwinienne, la recherche et le regroupement de tous les descendants d'un ancêtre commun; Alexandre n'est pas à Mussolini, Staline ou Hitler, via César et Napoléon, ce que le dryopithèque est au gorille. On peut cependant essayer de classer des faits sociaux ou culturels par familles, comme on le fait pour les familles de langues, en reconstituant leur filiation; on justifie, et on explique alors leur regroupement par l'histoire. On peut aussi former des catégories sur la base des similitudes entre des observations faites dans des contextes différents, isolés les uns des autres; le regroupement s'explique alors par la persistance d'une combinaison de traits remarquables, c'est-à-dire par une identité de structure. C'est l'idée de Durkheim: "Quand on est entré quelque peu en contact avec les phénomènes sociaux, on est (...) surpris de l'étonnante régularité avec laquelle ils se reproduisent dans les mêmes circonstances (...) Telle cérémonie nuptiale, purement symbolique à ce qu'il semble, comme l'enlèvement de la fiancée, se retrouve exactement partout où il existe un certain type familial (...) Les usages les plus bizarres, comme la couvade, le lévirat, l'exogamie, etc., s'observent chez les peuples les plus divers et sont symptomatiques d'un certain état social» (Durkheim 1960 [1894]: 94) ${ }^{39}$.

Il ne suffit pas de classer pour expliquer, même si l'on ne classe bien que si l'on a déjà commencé à expliquer. Il faut en effet distinguer entre les classifications purement descriptives, qui s'appuient sur des ressemblances de forme, c'està-dire apparentes et souvent trompeuses, et les classifications qui se fondent sur des ressemblances raisonnées, fonctionnelles ou généalogiques, qui sont déjà explicatives. La classification ne constitue sans doute qu'une étape préliminaire de la théorisation, mais c'est une étape nécessaire, un préliminaire indispensable (Bernard 1966 [1865] $)^{40}$. C'est en constituant progressivement et simultanément leurs catégories et leurs concepts à partir de l'observation, et en formant sur cette

${ }^{37}$ Plus les objets auxquels on s'intéresse sont matériels, et plus on peut les classer à la manière des naturalistes; c'est le cas par exemple des vestiges (poteries, bijoux, etc.) exhumés par les archéologues. C'est beaucoup plus difficile pour les entités abstraites auxquelles ont affaire les sociologues ou les ethnologues. On s'efforcera pourtant de définir celles-ci par des propriétés factuelles constatables, clairement énumérées et désignées, de les rapporter à des objets localisés, datés et situés, même si elles se répètent et se rencontrent dans des contextes différents.

${ }^{38}$ E. Mayr, Histoire de la biologie, op. cit., p. 172.

39 E. Durkheim, Les règles de la méthode sociologique, op. cit., p. 94.

${ }^{40}$ Pour C. Bernard, «la zoologie, donnant la description et la classification des espèces, n'est qu'une science d'observation qui sert de vestibule à la vraie science des animaux. Le zoologiste ne fait que cataloguer les animaux d'après les caractères extérieurs et intérieurs de forme». "L'évolution même des connaissances humaines veut que les sciences expérimentales soient le but, et cette évolution exige que les sciences de classification qui les précèdent perdent de leur importance à mesure que les sciences expérimentales se développent». Introduction à la médecine expérimentale, op. cit., pp. 157,204 
base, par inductions successives, des hypothèses de plus en plus générales, capables d'expliquer des ensembles de faits de plus en plus larges, que les sciences de l'homme collectif peuvent espérer produire des «théories bien fondées ». Ces hypothèses expliquent des phénomènes apparemment indépendants en montrant qu'ils ont des causes semblables, soit parce qu'ils obéissent à des lois identiques, invariantes sinon universelles, soit parce qu'ils sont l'aboutissement d'une même histoire, ou d'histoires entre lesquelles on peut trouver suffisamment de ressemblances. Elles s'énoncent sous la forme d'un principe qui constitue le fondement de la théorie, qui la résume et la désigne (par exemple, l'évolution). Ce principe est un principe explicatif, et non pas descriptif, comme c'est le cas de l'expression ou du nom générique par lesquels on désigne un ensemble de faits similaires au moyen du ou des traits distinctifs que ceux-ci ont en commun (comme par exemple «la famille patriarcale agnatique», ou «les religions de salut»). Mais un même principe peut aussi bien désigner ce qu'il faut expliquer (un ensemble de similitudes) que servir de principe d'explication; c'est le cas, on l'a vu, de l'habitus, ou encore du totémisme. En outre, dans nos disciplines, on a tendance à confondre les principes explicatifs avec les partis-pris interprétatifs caractéristiques des pseudo-théories. C'est le cas en particulier quand le concept principiel désigne un archétype à partir duquel on se propose de déduire logiquement les caractéristiques des variantes apparues dans des contextes séparés, sans avoir à reconstituer leur genèse ou leur diffusion. On n'a plus affaire à des catégories constituées en regroupant le plus grand nombre possible d'observations mais à des types idéaux obtenus par la sélection plus ou moins arbitraire d'un nombre réduit de caractères-clé. On court alors le risque de transformer ce type originel en principe universel, de croire par exemple que le totémisme, comme la lutte des classes, explique tout, ou, comme on dit, «rend compte» de tout (l'imprécision de cette formulation courante empêche de voir que par explication, on entend en fait interprétation). Il faut donc soumettre le plus possible au démenti de la réalité ces types qui par principe s'écartent de celle-ci, se demander dans quelle mesure ils correspondent aux observations dont on dispose. On trouve un bon exemple de cet examen critique chez M. Mauss, à propos des théories du sacrifice développées par R. Smith et ses prolongateurs, Jevons et Frazer:

Le grand défaut de ce système est de vouloir ramener les formes si multiples du sacrifice à l'unité d'un principe arbitrairement choisi. D'abord, l'universalité du totémisme, point de départ de toute la théorie, est un postulat. Le totémisme n'apparaît à l'état pur que dans quelques tribus isolées de l'Australie et de l'Amérique. Le mettre à la base de tous les cultes thériomorphiques, c'est faire une hypothèse, peut-être inutile, et qu'il est, en tout cas, impossible de vérifier (...) Mais acceptons un instant cette première hypothèse, quelque contestable qu'elle soit. La marche même de la démonstration est sujette à critique. Le point délicat de la doctrine est la succession historique et la dérivation logique que Smith prétend établir entre le sacrifice communiel et les autres types de sacrifice. Or rien n'est plus douteux. Tout essai de chronologie comparée des sacrifices arabes, hébreux ou autres qu'il étudiait, est fatalement ruineux. Les formes qui paraissent les plus simples ne sont connues que par des textes récents. Encore leur simplicité peutelle résulter de l'insuffisance des documents. En tout cas, elle n'implique aucune priorité. Si l'on s'en tient aux données de l'histoire et de l'ethnographie, on trouve partout le piaculum à côté de la communion. D'ailleurs ce terme vague de piaculum permet à Smith de décrire, sous la même rubrique et dans les mêmes termes, des purifications, des propitiations et des expiations, et c'est cette confusion qui l'empêche d'analyser le sacrifice 
expiatoire. Assurément, ces sacrifices sont généralement suivis d'une réconciliation avec le dieu; un repas sacrificiel, une aspersion de sang, une onction rétablissent l'alliance. Seulement, pour Smith, c'est dans ces rites communiels eux-mêmes que réside la vertu purificatrice de ces sortes de sacrifices; l'idée d'expiation est donc absorbée dans l'idée de communion». Smith écarte les faits contrariants (encore les mentionne-t-il): «Sans doute, il constate, dans quelques formes extrêmes ou simplifiées, quelque chose qu'il n'ose pas rattacher à la communion, une sorte d'exorcisme, d'expulsion d'un caractère mauvais. Mais, suivant lui, ce sont des procédés magiques qui n'ont rien de sacrificiel et il explique avec beaucoup d'érudition et d'ingéniosité leur introduction tardive dans le mécanisme du sacrifice. Or c'est précisément ce que nous ne pouvons accorder (Mauss 1969, I: 193$)^{41}$.

\section{SOCIOLOGIE ET HISTOIRE}

L'influence de la philosophie pousse en effet l'anthropologie et, à sa suite, la sociologie à généraliser trop vite, à opter pour une épistémologie implicite qui dévalorise l'observation, tourne le dos à la conceptualisation progressive et exclut la reconstitution narrative des enchaînements et des hasards historiques. Vouloir retrouver au plus vite les structures universelles de l'esprit humain, comme le propose l'anthropologie structurale, c'est risquer de revenir à l'homme universel abstrait des «humanités» et de l'humanisme ${ }^{42}$, et de ramener une fois encore à l'unité de la «nature humaine» la diversité sociale, culturelle ou historique qui constitue l'objet même des sciences de l'homme collectif.

Peut-être, un jour, la sociologie saura-t-elle déterminer avec précision ce qu'il y a de commun chez les individus de tous les groupes humains. Présentement, une tâche plus modeste s'impose. Il faut analyser d'abord, avec le plus de rigueur possible, la riche diversité qui s'offre à l'observation, et que nous n'avons pas le moyen, aujourd'hui, de ramener à l'unité. (...) L'histoire et l'anthropologie nous mettent en présence d'une réalité infiniment variée et complexe, et nous sommes bien forcés de reconnaître que nous n'en obtiendrons la connaissance qu'au prix de longs efforts, méthodiques et collectifs, comme lorsqu'il s'agit de la nature donnée à nos sens. Aussitôt que nous considérons des sociétés différentes de celle où tout nous paraît clair, parce que tout nous y est familier, nous rencontrons à chaque pas des problèmes que nous sommes incapables de résoudre par le simple bon sens, aidé seulement de la réflexion et de la connaissance courante de la 'nature humaine'. Les faits qui nous déconcertent obéissent sans doute à des lois: mais quelles sont-elles? Nous ne saurions le deviner. La réalité sociale offre, en un sens, plus de difficultés que le monde physique à la recherche scientifique, car, même en supposant connues les lois statiques, l'état d'une société, à moment donné, n'est jamais intelligible que par l'évolution antérieure dont il est le point d'aboutissement actuel; et combien sont rares les cas où la connaissance historique de ce passé est assez complète et assez sûre pour que rien d'indispensable ne fasse défaut! Aussi bien, est-ce là une raison de plus pour ne jamais s'écarter d'une méthode scrupuleusement objective, et pour nous attendre à ce qu'ici, comme dans la science de la nature physique, le vraisemblable ne soit pas souvent le vrai (Lévy-Bruhl 1923 [1903]: 79) ${ }^{43}$.

\footnotetext{
${ }^{41}$ M. Mauss, Euvres, op. cit., t. I, pp. 193 sq.

${ }^{42}$ E. Durkheim, L'évolution pédagogique en France, op. cit., pp. 312-314.

${ }^{43}$ L. Lévy-Bruhl, La morale et la science des mours, op. cit., p. 79.
} 
En passant en revue des cultures séparées dans le temps et dans l'espace, on cherche alors non des vestiges, des traces d'une origine commune, mais la marque d'une structure intemporelle, identique et constante. La formalisation des récits mythiques repose sur la recherche et la mise en évidence des traits que ceux-ci ont en commun; au lieu d'être attentif et de faire droit aux différences qu'ils présentent, on les élimine, au lieu de chercher à dégager les variations auxquelles ces différences peuvent correspondre, on recherche des invariants. L'anthropologie structurale ignore la dimension chronologique des faits de culture au profit d'une démarche typologique; elle néglige par principe les filiations et les échanges, les processus qui assurent la transmission, la diffusion et la transformation des mythes, des rituels ou des systèmes de parenté. Elle invite ainsi les sciences de l'homme à théoriser sur le modèle des sciences nomothétiques; mais en même temps elle les renvoie du côté des savoirs herméneutiques en faisant de l'observation matière, affaire d'interprétation. La structure dont on veut et dont on croit détecter la présence dans un matériel ethnographique (ou aussi bien dans des documents ou dans des interviews) a tendance en effet à jouer le rôle d'une clé interprétative universelle et infaillible, que sa circularité empêche de prendre en défaut: chacun des éléments, chacune des observations dont se compose le matériel «renvoie» à la structure sous-jacente, confirme sa présence; la structure «rend compte» en retour de chacun des éléments dont se compose le matériel (sur le «circulus herméneutique», cf. supra, chapitre 2,3).

L'antagonisme entre l'inspiration philosophique et le point de vue historique se voit particulièrement bien dans l'opposition entre les structuralismes respectifs de C. Lévi-Strauss et de G. Dumézil, qui entend se borner «à établir des faits - des faits du second degré, des prototypes probables, déduits de la comparaison des faits du premier degré, des faits constatés -, mais en tout cas, des faits particuliers » (Dumézil 1987: 121) ${ }^{44}$. Dans Anthropologie structurale, C. Lévi-Strauss dit avoir «emprunté la notion de structure à Marx et à Engels » (plutôt qu'à Radcliffe-Brown, dont il se démarque dans le même ouvrage en refusant le fonctionnalisme, c'est-à-dire le couplage de la structure et de la fonction, qu'il qualifie de «forme primaire de structuralisme»). Les ouvrages qui ont le plus contribué à populariser sa pensée - Tristes tropiques (1955) et La Pensée sauvage (1962) associent étroitement les récits ethnographiques, les réflexions personnelles à caractère autobiographique, les questions de méthode et les questions philosophiques et morales. Le primat que C. Lévi-Strauss accorde à la généralisation sur

${ }^{44}$ G. Dumézil, Entretiens avec Didier Eribon, Paris, Gallimard, Folio, 1987, p. 121.

«G.D.: Chacun de nous est inévitablement marqué par sa formation scolaire, par les études qui l'ont préparé à ses examens, à ses concours. Cela nous conditionne: orientation, mais aussi, limites. Lévi-Strauss a grandi dans la philosophie, moi dans la philologie. Je ne pose donc pas comme lui les grands problèmes, même si je les entrevois à l'horizon. Bien qu'on parle quelquefois de la théorie dumézilienne, je ne suis pas théoricien, ni 'dumézilien' (...)

D.E.: Vous ne voulez pas qu'on quitte le champ des faits?

G.D. : On a parfaitement le droit d'en sortir. Mais il faut alors savoir qu'on fait de la poésie, de la philosophie, c'est-à-dire du rêve. Pourquoi ne rêverait-on pas?

D.E. : Vous affirmez souvent que vous ne voulez pas délivrer de message.

G.D.: Je n'ai jamais envisagé qu'on puisse tirer de mon travail un système philosophique en dehors de mon petit domaine». 
la comparaison caractérise en propre la démarche philosophique: «En ethnologie comme en linguistique (...) ce n'est pas la comparaison qui fonde la généralisation, mais le contraire. Si (...) l'activité inconsciente de l'esprit consiste à imposer des formes à un contenu, et si ces formes sont fondamentalement les mêmes pour tous les esprits (...), il faut et il suffit d'atteindre la structure inconsciente, sousjacente à chaque institution ou à chaque coutume, pour obtenir un principe d'interprétation valide pour d'autres institutions et d'autres coutumes » (Lévi-Strauss 1949: 363-391) $)^{45}$.

Pour théoriser, la sociologie doit au contraire replacer les faits sociaux dans le temps. L'exploration du passé élargit et diversifie l'aire de recherche où le sociologue peut rencontrer les faits contrariants qui font obstacle aux généralisations hâtives et préviennent la dérive interprétative. La mise en perspective historique accroît ainsi les occasions de démenti qui s'offrent aux conjectures des sociologues. En outre le caractère événementiel des faits historiques, le fait qu'ils ne se reproduisent pas et qu'ils ne se répètent jamais à l'identique découragent les comparaisons et les rapprochements hâtifs. Leur individualité forte limite les risques de schématisation abusive, oppose une résistance particulière à la modélisation arbitraire et orientée qui ne retient que les faits capables d'illustrer et de soutenir un parti-pris interprétatif. On peut sans doute nier la pertinence d'un événement historique, le tenir pour un accident négligeable et inessentiel; mais il est plus difficile de nier son existence de fait. Les faits historiques sont des événements singuliers, désignés par des noms propres; cette singularité leur confère une réalité qui s'impose et résiste aux tentatives de théorisation avec la force réfutatrice des evidences matérielles. Le sociologue qui travaille sur des données historiques est ainsi amené à ne pas s'en tenir à une définition purement logique de la causalité et de la preuve. La datation, qui fait la spécificité du fait historique, ou la dimension historique des faits sociaux, est ambivalente: dater un événement, c'est à la fois le positionner, le localiser dans le temps, et l'insérer dans une chaîne temporelle qui est aussi un enchaînement causal; c'est postuler que les phénomènes sociaux sont le résultat d'une histoire, et qu'on ne peut les expliquer qu'en reconstituant dans le détail la succession des processus, des micro-histoires dont cette histoire est faite, et dont leur état présent est l'aboutissement.

Se tourner vers le passé, pour un sociologue, c'est aussi rencontrer les problèmes que pose l'explication par l'origine. Durkheim voit dans la forme élémentaire (par exemple de la vie religieuse) la forme simple et abstraite, dont les manifestations que l'on observe à différentes époques et dans différents lieux sont des dérivés et des variantes. D'un côté, c'est une démarche historique, narrative: on va dans le passé rechercher l'ancêtre commun, on remonte le temps et on parcourt l'espace, en gardant l'idée de transmission, d'enchaînement et de diffusion. Mais de l'autre, à l'opposé, c'est une démarche typologique et modélisatrice: à la fois type primitif et type de principe, défini par la réunion «d'un petit nombre» de «caractères particulièrement essentiels », l'archétype est l'abstraction à laquelle on cherche à réduire la diversité culturelle et historique des contenus concrets. Peut-on et doit-on associer ces deux démarches, s'efforcer à la fois

${ }^{45}$ C. Lévi-Strauss, «Histoire et ethnologie», Revue de Métaphysique et de Morale, 54ªnnée, n 3-4, 1949, pp. 363-391 (repris in C. Lévi-Strauss, Anthropologie structurale, Paris, Plon, 1958). 
d'historiser la sociologie et de sociologiser l'histoire? Modéliser, c'est schématiser, simplifier, et donc poser en principe que la complexité des phénomènes est une illusion qu'il faut travailler à dissiper; c'est assimiler la clarification à la simplification, ou du moins faire de la seconde la condition de la première. L'épistémologie narrative et probabiliste qui convient aux sciences historiques ne réduit pas la clarification à la simplification. Mais elle n'exige nullement qu'on renonce à la clarification; elle oblige au contraire à démêler l'enchevêtrement des causes et des effets, à dénouer les processus les plus embrouillés, à raconter clairement les histoires les plus obscures. Elle n'exige pas davantage qu'on renonce à simplifier; pour reconnaître la complexité réelle qui caractérise et distingue l'ordre humain, il faut la distinguer de leur complexité apparente et illusoire, qu'il faut écarter.

Le sociologue peut sans doute adopter le point de vue de l'anthropologue et lui emprunter ses concepts; c'est le cas par exemple quand, au lieu ou en plus de mettre les pratiques alimentaires en relation avec le système des ressources ou l'éducation, on les envisage comme un cas particulier de l'échange, à la lumière de notions telles que le don, l'honneur, le calcul ou le sacrifice. On se donne ainsi le moyen de mettre à distance et en question des pratiques qui semblent aller de soi. L'approche ethnologique aide à la relativisation des goûts et des usages, active le sentiment de l'arbitraire dont l'esprit sociologique est animé; en posant en principe que les faits de civilisation sont des traits de culture, que les manières de table, les raffinements, les politesses, les cérémonies sont (et ne sont que) des faux-semblants et des déguisements, des accessoires de la comédie sociale, elle renoue avec la tradition irrévérencieuse et satirique dont la sociologie est issue. Mais cette capacité critique se paye d'une réduction de l'ordre sociologique à l'ordre dit anthropologique. Le sociologue qui adopte systématiquement le point de vue de l'ethnologue est conduit à considérer les faits sociaux comme des apparences. Il s'expose ainsi à manquer son objet en manquant le niveau de complexité qui caractérise celui-ci; d'un point de vue purement ethnologique, les formations sociales ne sont que des élaborations, des complications à partir d'un socle primitif, ou plutôt primordial. L'approche anthropologique est tentante, parce qu'elle semble offrir au sociologue un raccourci explicatif (c'est aussi le cas lorsque celui-ci, adoptant le point de vue de l'économiste, applique systématiquement l'axiomatique benthamienne et le raisonnement utilitariste aux comportements les plus «désintéressés»). Mais l'explication consiste alors à traiter les observations comme des signes qui «renvoient» à une réalité profonde, structure, nature ou essence. Sous peine de succomber à l'obsession interprétative on suivra donc l'exemple de Mauss; on mettra les rapprochements que l'on opère à l'épreuve des faits et de la reconstitution historique, et ce d'autant plus qu'ils sont plus évocateurs et plus suggestifs. On jouera pour ainsi dire la chronologie et la classification contre la typologie; au lieu de rattacher directement et hâtivement les observations dont on dispose à un archétype, on s'efforcera de les regrouper en familles, et de dégager ainsi progressivement des histoires et des cas typiques. Peut-être parviendra-t-on ainsi à rassembler des pratiques apparemment hétérogènes, en dégageant les éléments archaïques qu'elles ont en commun. 